\title{
The costs of achieving climate targets and the sources of uncertainty
}

\author{
D. P. van Vuuren $\mathbb{D}^{1,2}$, Kaj-Ivar van der Wijst ${ }^{1}$ 1,2,3, Stijn Marsman ${ }^{\circledR 1}$, Maarten van den Berg ${ }^{1}$, \\ Andries F. Hof ${ }^{1,2}$ and Chris D. Jones (1D) 4
}

Effective climate policy requires information from various scientific disciplines. Here, we construct a metamodel from climate and integrated assessment models that assesses the emissions budget, costs and uncertainty sources of achieving temperature targets. By calibrating to the model-based literature range, the metamodel goes beyond the parametric uncertainty of individual models. The resulting median estimates for the cumulative abatement costs (at $5 \%$ discount rate) for $2{ }^{\circ} \mathrm{C}$ and $1.5^{\circ} \mathrm{C}$ targets are around US\$15 trillion and US\$30 trillion, but estimates vary over a wide range (US\$10-100 trillion for the $1.5^{\circ} \mathrm{C}$ target). The sources determining this uncertainty depend on the climate target stringency. Climate system uncertainty dominates at high warming levels, but uncertainty in emissions reductions costs dominates for the Paris Agreement targets. In fact, costs differences between different socio-economic development paths can be larger than the difference in median estimates for the $2^{\circ} \mathrm{C}$ and $1.5^{\circ} \mathrm{C}$ targets. This simple metamodel helps to explore implications of scenario uncertainty and identify research priorities.

T he costs of achieving climate targets depend on a number of uncertain factors, including the relationships between GHG emissions and radiative forcing, temperature change and the costs of reducing $\mathrm{CO}_{2}$ and other GHGs ${ }^{1}$. Many scientific assessments and (multi-)model studies have been published that cover certain aspects of these relationships (for example, socio-economic $^{2-5}$ or biophysical ${ }^{6-8}$ ). Together, they provide a number of key insights, such as the near-linear relationship between cumulative $\mathrm{CO}_{2}$ emissions and global mean temperature and the rapidly increasing abatement costs for more stringent climate targets. These relationships have been quantified by multiple models, covering a wide range of methods and assumptions. Still, the uncertainty space currently covered in model studies represents only part of the total uncertainty space, given the necessary model simplifications and the limited number of (scenario) uncertainties that can be explored. There is an ongoing discussion about the relevance of considering a wider range of scenarios going beyond the space currently covered by models and the need for scenario updates ${ }^{9-11}$. Nonetheless, one can reasonably assume that the model ranges represent a relevant part of the uncertainty range (based on a combination of theory, model calibration and expert elicitation) and they also form a key quantitative input in the assessments of the IPCC. Here, we introduce a metamodel based on the key model outcomes in different parts of the IPCC assessment describing the relationship between temperature targets, carbon budgets and mitigation costs, including the associated uncertainty ranges. The strength of this model is its transparency and its ability to use the full model uncertainty range in the literature, covering a range of models, approaches and scenario assumptions. As such, it goes beyond the parametric uncertainty captured by single models that also describe this topic (for example, refs. ${ }^{12,13}$ ).

\section{A metamodel of mitigation costs}

The elements used for constructing the metamodel, including the 5-95th percentile range of output in the literature, are shown in Fig. 1.
The first element is the transient climate response to cumulative $\mathrm{CO}_{2}$ emissions (TCRE), which shows the relationship between cumulative $\mathrm{CO}_{2}$ emissions and temperature change (Fig. 1a). This emergent property of Earth system model (ESM) simulations implies that each temperature target can be translated relatively easily into a carbon budget, given a specific choice of how to deal with uncertainty ${ }^{14}$. The TCRE relationship has been reported for runs with only $\mathrm{CO}_{2}$ forcing, and also for scenarios including non$\mathrm{CO}_{2}$ gases. The TCRE is a function of various underlying sources of uncertainty in the physical system. For example, Gillett et al. ${ }^{15}$ show how TCRE can be constructed from climate sensitivity and carbon cycle components. Others ${ }^{16}$ have tried to estimate the individual role of the physical climate response and biogeochemical feedbacks, suggesting that both represent approximately half the uncertainty in the TCRE. Millar et al. ${ }^{8}$ published a slightly revised method to estimate carbon budgets using the TCRE, by shifting the outcomes of ESM simulations for the base year using the historically observed temperature and emissions data. This method is also used here, using an estimate for 2010 warming based on a range of historical time series and methods showing a range of $0.76-1.06^{\circ} \mathrm{C}$ compared with the pre-industrial period ${ }^{17}$.

Non- $\mathrm{CO}_{2}$ GHGs also influence future temperature. In the scenario literature, a correlation between non- $\mathrm{CO}_{2}$ GHG forcing and cumulative $\mathrm{CO}_{2}$ emissions can be found, as both partly originate from the same sources and are influenced by the strength of climate policy in the scenarios (nearly all scenarios assume the same equivalent price for both $\mathrm{CO}_{2}$ and non- $\mathrm{CO}_{2}$ ). We estimated the temperature impact of non- $\mathrm{CO}_{2}$ GHGs to capture this as a separate factor. This is done by using data from the Shared Socioeconomic Pathways (SSPs) database ${ }^{4,5}$. The reason for using this database is that the SSPs explicitly paid attention to the range of assumptions for non- $\mathrm{CO}_{2}$ gases. In Fig. 1, we compare the SSP data with the scenario data of the IPCC Special Report on Global Warming of $1.5^{\circ} \mathrm{C}$ $(\mathrm{SR} 1.5)^{18}$, showing that the SSP range covers the wider model literature range very well.

\footnotetext{
'PBL Netherlands Environmental Assessment Agency, the Hague, the Netherlands. ${ }^{2}$ Copernicus Institute of Sustainable Development, Utrecht University, Utrecht, the Netherlands. ${ }^{3}$ Mathematical Institute, Utrecht University, Utrecht, the Netherlands. ${ }^{4}$ Met Office Hadley Centre, Exeter, UK. 凶e-mail: Detlef.vanVuuren@pbl.nl
} 
a
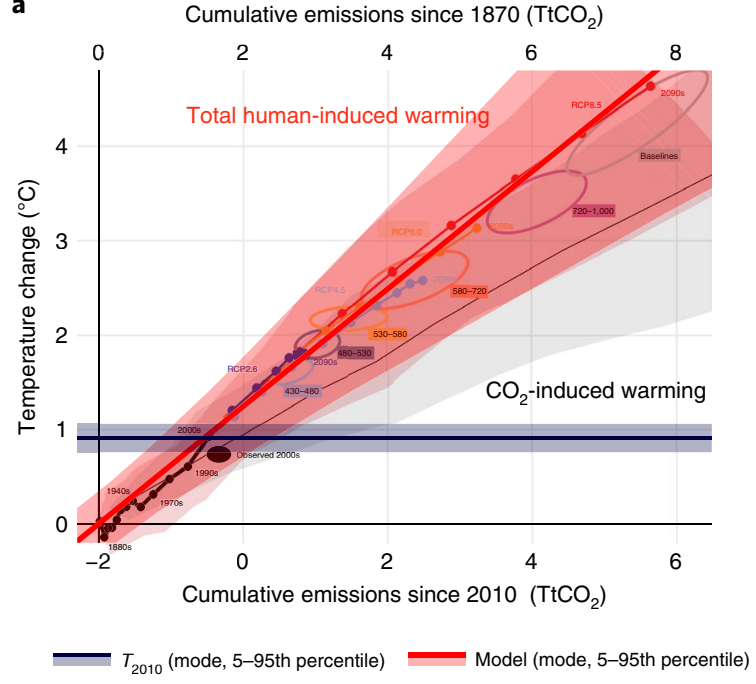

C

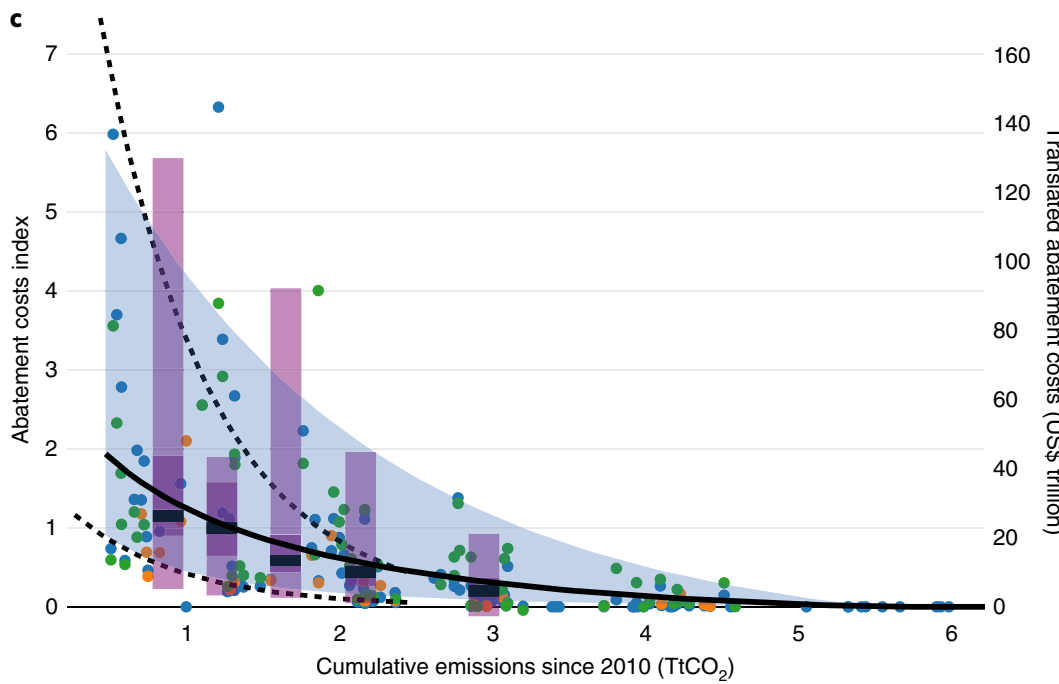

b

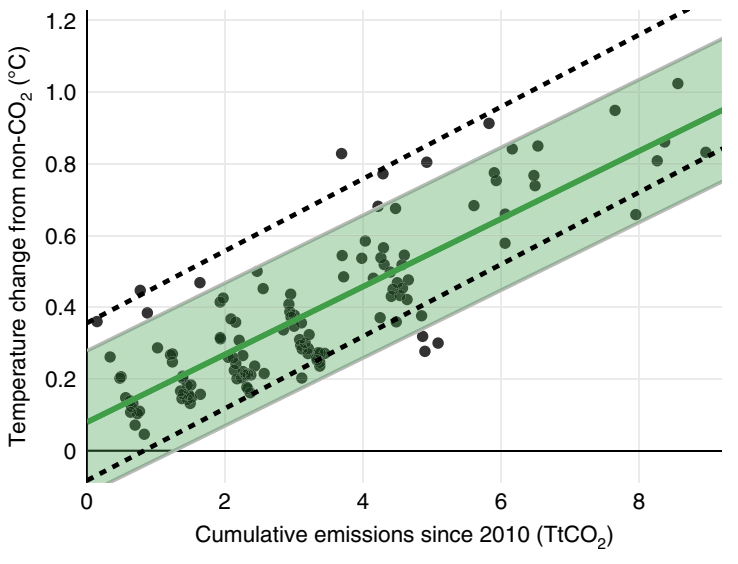

Model (mode, 5-95th percentile) $\quad$..... SR1.5 (5-95th percentile)

- SSP data

Fig. 1 | Key relationships in the literature relevant to the costs of achieving climate targets. a-c, The relationship between cumulative $\mathrm{CO}_{2}$ emissions and temperature (TCRE), based on the 2010 estimate for global mean temperature ${ }^{17}$ and the relationship found by IPCC AR5 (ref. ${ }^{29}$; a), the relationship between cumulative $\mathrm{CO}_{2}$ emissions and forcing of non- $\mathrm{CO}_{2} \mathrm{GHGs}$, as reported in the SSP and SR1.5 databases ${ }^{4,5,18}$ (b) and the relationship between cumulative $\mathrm{CO}_{2}$ emissions and the cost index (relative costs for various metrics relative to the average costs of achieving an emission target of $1.6 \mathrm{TtCO}$ over the 2010-2100 period), based on the SSP database $\mathrm{e}^{4,5}, \mathrm{AR} 5$ ( ref. $^{2}$ ) and SR1.5 (c) (see Supplementary Information). Note that the SR1.5 cost curve is truncated, since the corresponding database mainly focuses on low emission scenarios. In all cases, the mean relationship as used in the model and the 5-95th percentile range is shown. RCP, representative concentration pathway.

Another key determinant of total mitigation costs is the relationship between the costs and the stringency of climate policy. In the literature, a range of models and metrics is used to express the costs of climate policy, including macroeconomic indicators such as consumption loss, and more direct cost estimates such as abatement $\operatorname{costs}^{2,19}$. For our metamodel, we used this information by converting the various cost estimates in the relative costs compared to those for reaching a $1.6 \mathrm{TtCO}_{2}$ carbon budget (left $y$ axis, see Methods). For ease of interpretation, the index is also converted to one of the underlying metrics, that is, net present value of consumption loss (right $y$ axis). The results show for different metrics a very similar exponential increase with the stringency of the emission target, here represented by cumulative $\mathrm{CO}_{2}$ emissions (Fig. 1c). This finding is consistent with the general assumption of increasing marginal costs as a function of the GHG reduction rate ${ }^{2,19}$ (only a few studies show opposite results ${ }^{20}$ ). We again use the more recent data of the SSPs for the model, as other databases, such as the one for the IPCC Fifth Assessment Report (AR5), represent only part of the relevant range for climate targets ${ }^{4,5}$. Nonetheless, Fig. 1c compares the range of the SSPs with the literature overviews in AR5 (ref. ${ }^{2}$ ) and SR1.5 (ref. ${ }^{18}$ ), showing similar ranges (the SR1.5 data are not representative for high carbon budgets due to the low number of scenarios included). The range in abatement costs for similar cumulative $\mathrm{CO}_{2}$ emission targets is substantial. Important factors contributing to this range are differences in (1) assumptions on socio-economic and technology development, (2) the feasibility of upscaling critical low-carbon technologies, (3) the extent to which climate policies induce further economic impacts, (4) the type of policies applied and (5) the ability to reduce emissions in hard-to-abate sectors ${ }^{2,4,21,22}$.

Based on the results for mitigation costs shown in Fig. 1, it is possible to derive the following simple metamodel on mitigation costs:

$$
\text { Costs }=a+b \times e^{-c \int_{2010}^{2100} \operatorname{emis}(t) \mathrm{d} t}=a+b \times e^{-c\left(T_{\text {target }}-T_{2010}-\sigma_{\mathrm{nCO}_{2}}\right) / \mathrm{TCRE}}
$$



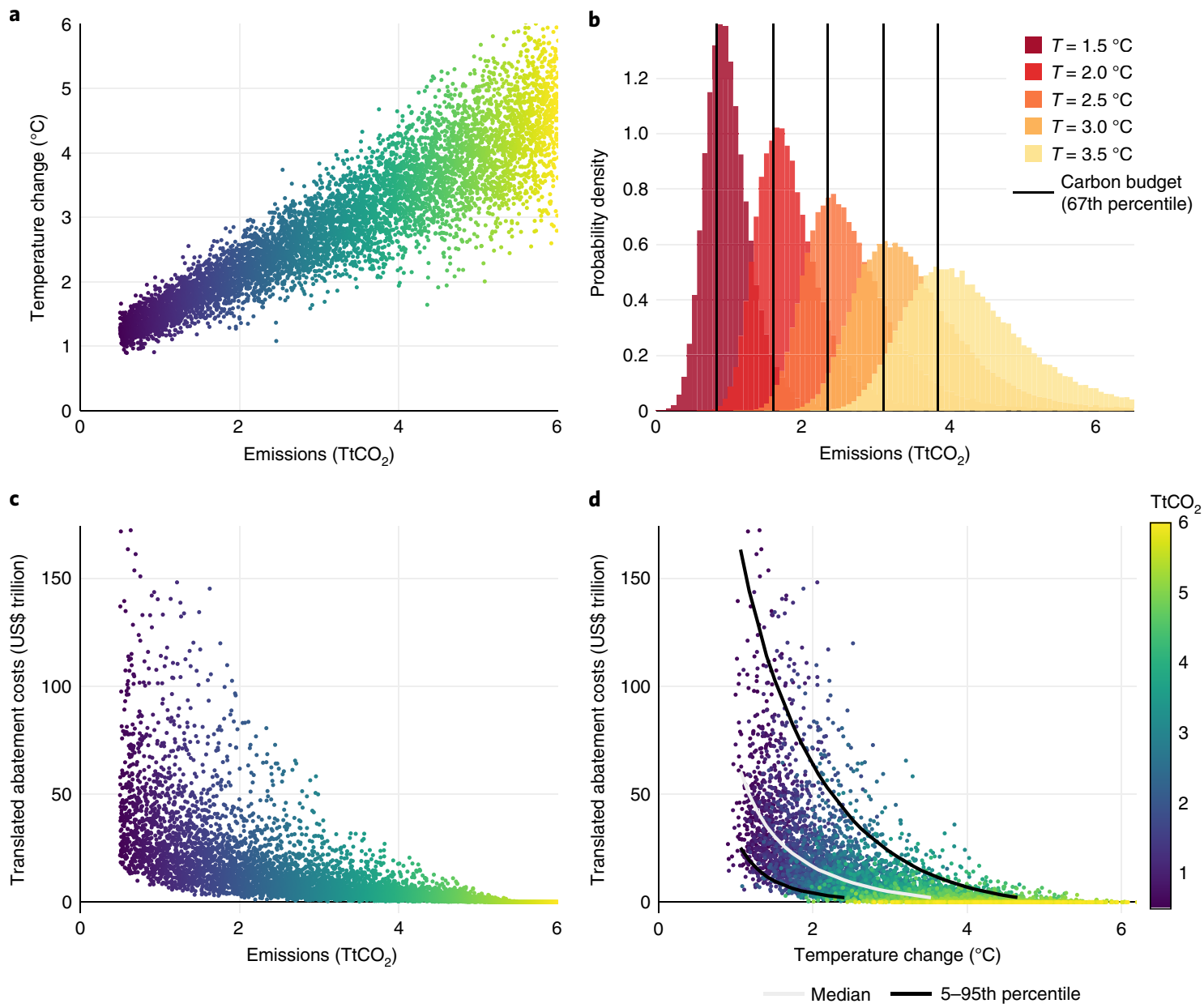

Fig. 2 | Application of the metamodel. a-d, Temperature change as a function of cumulative emissions (a), the normalized histogram of carbon budgets for the different temperature targets (b), abatement costs as a function of cumulative emissions (c) and abatement costs as a function of temperature targets (d). The costs are expressed as the net present value of abatement costs, using a 5\% discount rate. In the Monte Carlo analysis, 6,500 data points were drawn.

where the parameters $a, b$ and $c$ are determined using the SSP cost data; $T_{\text {target }}$ the target temperature; $T_{2010}$, the temperature in 2010; and $\sigma_{\mathrm{n} C \mathrm{CO}_{2}}$, the contribution of non- $\mathrm{CO}_{2}$ gases. The values of each of these factors and the associated range (5-95th percentile) can be directly derived from the panels of Fig. 1 (see Methods), allowing a Monte Carlo analysis (assuming a log-normal distribution) to estimate the costs of achieving different climate targets with associated uncertainties. Consistent with most long-term scenarios, we focused on the period up to 2100 . It should be noted that this model describes a time-independent problem (assuming that the underlying time dynamics are already captured in the individual factors, see Limitations of the metamodel).

\section{Costs and the contribution of uncertainty}

Based on the model results (Fig. 2a), carbon budgets can be derived for the various temperature targets (Fig. 2b). The derived budgets are $837 \mathrm{GtCO}_{2}$ (5-95th percentile: $\left.539-1,580\right)$ and $1,600 \mathrm{GtCO}_{2}$ $(1,219-2,700)$ for the $1.5^{\circ} \mathrm{C}$ and $2{ }^{\circ} \mathrm{C}$ temperature targets, respectively. The results compare well to those of IPCC SR1.5 $\left(\right.$ ref. ${ }^{18}$ ), that is, $746 \mathrm{GtCO}_{2}$ and $1,496 \mathrm{GtCO}_{2}$ for the $2010-2100$ period: the difference is mostly caused by the choice in the IPCC report to account for the additional uncertainty beyond model results (see Methods). Other differences come from different treatments of uncertainty in temperature and non- $\mathrm{CO}_{2}$ emissions. The results also depend on the assumptions regarding the form of the uncertainty distribution on the TCRE (see also ref. ${ }^{23}$ ). Similar to the IPCC SR1.5 budgets, the climate feedbacks not covered in the models used in the fifth round of the Coupled Model Intercomparison Project (CMIP5) could lead to a reduction of $100 \mathrm{GtCO}_{2}$ in the budgets mentioned here ${ }^{18}$. The model finally shows how abatement costs increase for more stringent emission (Fig. 2c) and temperature targets (Fig. 2d). Costs are low for high targets, but increase rapidly for stringent targets. For the Paris Agreement targets (limiting the increase in global mean temperature to well below $2^{\circ} \mathrm{C}$ and pursuing efforts to limit it to $1.5^{\circ} \mathrm{C}$ ) this leads to median abatement cost estimates (in terms of present value using a $5 \%$ discount rate) of US $\$ 16.4$ trillion for $2^{\circ} \mathrm{C}$ and US $\$ 30.5$ trillion for $1.5^{\circ} \mathrm{C}$, but with a 5-95th percentile range of US\$4.1-63.4 and US\$9.8-103.5 trillion, respectively. The low side of these ranges is based on favourable socio-economic development for mitigation, low-cost models, a favourable TCRE and relatively low non- $\mathrm{CO}_{2}$ gases, while on the high side the opposite conditions are true. The impact of socio-economic development can be shown by breaking up the costs range in the SSP database (Fig. 1c) for different SSPs, grouped by their challenge to mitigation (Fig. 3b). Interestingly, the reported range in abatement costs caused by all of these factors is a factor 10 (Fig. 2d), which is much larger than the difference in mean cost estimate between the $1.5^{\circ} \mathrm{C}$ and $2{ }^{\circ} \mathrm{C}$ target (a factor 2).

The unique contribution of the metamodel is that it allows one to estimate an uncertainty range consistent with the model literature. 
a

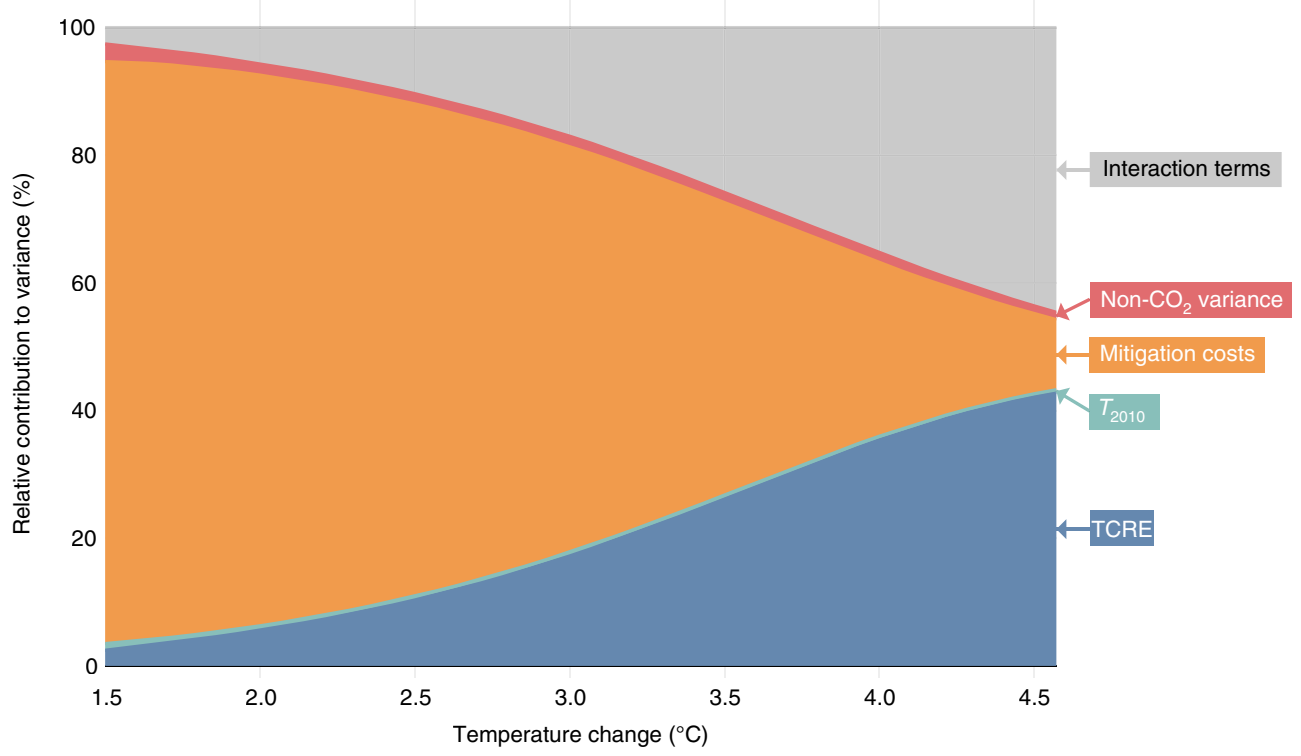

b

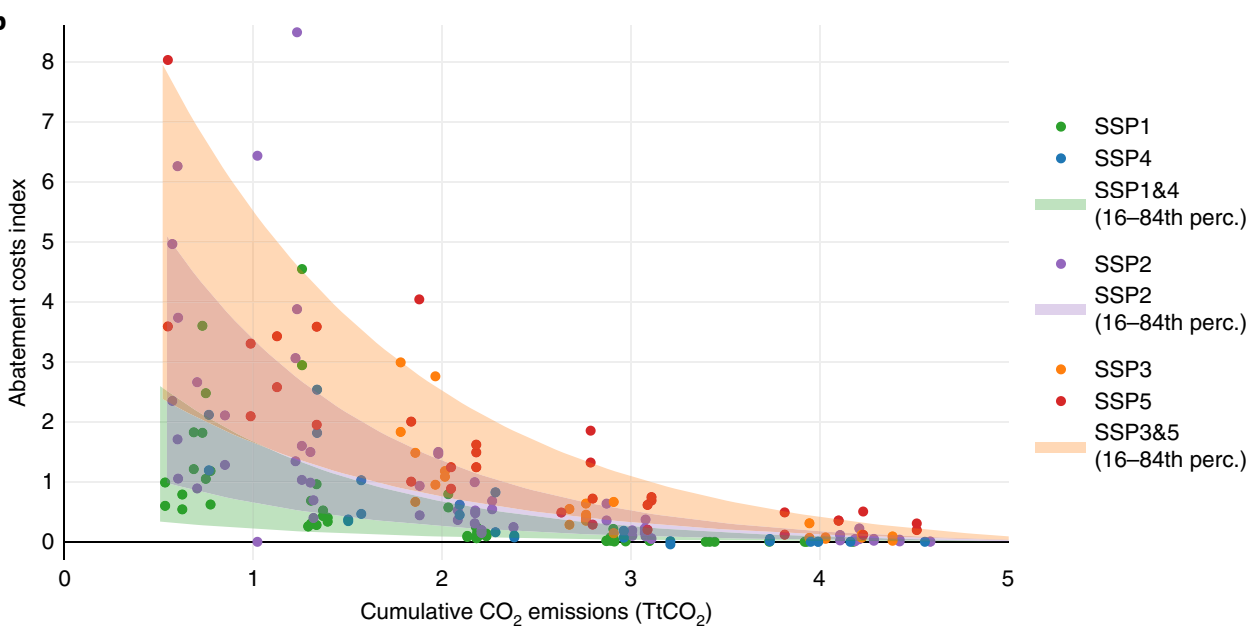

Fig. 3 | The relative contribution of single factors in the overall variance in mitigation costs. $\mathbf{a}, \mathbf{b}$, Contribution is shown as a function of the temperature target (a) and the uncertainty in abatement costs as a function of cumulative $\mathrm{CO}_{2}$ emissions, shown as a function of different socio-economic development assumptions (SSPs) grouped by challenges for mitigation (less difficult for SSP1 and SSP4; more difficult for SSP3 and SSP5) based on the SSP database (b).

By calculating the relative variance originating from the uncertainty of each individual factor, it is also possible to determine which uncertainties contribute most to the uncertainty range in abatement costs (Fig. 3). We focus here on the direct contribution of each factor (ignoring the interaction terms, see Supplementary Fig. 11; the contribution of interaction terms is larger for high temperature targets but trends are similar as for the direct contributions). For relatively high temperature targets (for example, between around 2.5 and $3^{\circ} \mathrm{C}$ ), an important part of the uncertainty about costs is associated with that on TCRE, that is, our limited understanding of the climate system and carbon cycle. For stringent targets, the dominating factor is the uncertainty in mitigation costs. Although the contribution of non- $\mathrm{CO}_{2}$ gas and 2010 temperature uncertainty also become increasingly important for stringent climate targets for carbon budgets, regarding costs this trend is swamped by the increasing mitigation cost uncertainty. The increasing importance of mitigation cost uncertainties with more ambitious climate targets is consistent with the philosophical understanding that a $3^{\circ} \mathrm{C}$ warmer climate would represent an energy economy that is relatively close to that of today and a very different climate system, while a $1.5^{\circ} \mathrm{C}$ warmer climate would represent a very different energy economy and a climate system that is closer to today's system. A few earlier studies have highlighted the dominant role of socio-economic uncertainty or choices for stringent targets ${ }^{13,24-26}$. These studies, however, focused on the sensitivity of a specific model. Here, we confirm this finding based on the literature ranges of climate and integrated assessment model results, also relating the dynamics to tangible parameters currently used in the literature, such as the TCRE.

\section{Limitations of the metamodel}

While the strength of the metamodel is the representation of different forms of uncertainty, the analysis also has a number of limitations. The most important is that the model is obviously limited by the available literature on which it is based. Earlier, we discussed how current model results only cover part of the real-world uncertainty. For instance, nearly all mitigation scenarios in the literature assume optimal climate policies. Non-optimal strategies (for example, delayed mitigation) could lead to higher costs or a different 
functional relationship. At the same time, under-representation of induced technical change could lead to lower costs. The scenarios also do not cover the impact of climate change on socio-economic development. One other aspect of this is that the quantitative literature focuses only on the aspects of climate policy that can easily be quantified. Clearly, there are other factors contributing to the uncertainty of costs, such as the assumed level of good governance and processes of social learning. Another limitation is that the mitigation problem in the metamodel is assumed to be time-independent (see equation (1)). Partly, the time-dependency is captured implicitly in the mitigation cost estimates, such as the fact that more stringent climate targets will require early action (also associated with different technology dynamics). Other time-dependent factors, however, are ignored, particularly for short-lived GHGs and non-optimal emission profiles. The model also aggregated all emissions (positive or negative) over time. While real 'expert' models can explore the impacts of dynamic factors, the results from the metamodel need to be used with more care if the timing of emissions becomes more dominant, that is, for very stringent targets such as those below $1.5^{\circ} \mathrm{C}$. Finally, the model does not capture the costs of climate impacts or adaptation costs (although, in principle, these could be included), which could clearly also influence both the shape of costs and the contribution to uncertainty ${ }^{25,27}$.

\section{Implications for research and assessment}

Despite the limitations, the metamodel offers a whole-system assessment, taking into account the state-of-the-art from key climate research communities. The metamodel does not rely on the particular characteristics of a single model and can be easily updated with new results (for example, CMIP6 model results or new economic model runs). Finally, it can also be easily extended; for instance, by distinguishing the climate sensitivity and carbon cycle component in TCRE.

The model's outcome that large uncertainty ranges are related to the carbon budgets and mitigation costs emphasizes the need to be careful with precise numbers and to focus in communication more on the uncertainty ranges ${ }^{28}$. Clearly, it is also helpful to reduce the uncertainty on the information, as this reduces the risk of making wrong decisions. If the costs of achieving the $1.5^{\circ} \mathrm{C}$ target are as low as US $\$ 10$ trillion, it would probably pay to implement stringent policies to avoid the risks related to higher temperature targets. If, in contrast, the costs are as high as US\$100 trillion or more, incentivizing sufficient investment flows will be more difficult (and it might be important to invest more in adaptation strategies). The finding that, for climate targets associated with the Paris Agreement, the abatement cost uncertainty is largely driven by uncertainty in the 'economic' system rather than natural system is of key importance. This implies that, if one aims to reduce the uncertainty range, it is much more important to reduce the 'economic uncertainty' rather than the 'climate science uncertainty'. This emphasizes the need to invest in research on mitigation costs. Such research may focus on the critical factors mentioned before: (1) socio-economic and technology development, (2) the feasibility to upscale critical technologies, (3) induced economic impacts, (4) the effectiveness of policy instruments and (5) the reductions in hard-to-abate sectors ${ }^{2,421,22}$. Moreover, the fact that the uncertainty in socio-economic factors can have a stronger impact on the mitigation costs than the difference between the 1.5 and $2{ }^{\circ} \mathrm{C}$ target emphasizes the importance on focusing on development policies beyond strict climate policy and the need to consider multiple scenarios in assessments. In the future, the simple metamodel can also be used to explore other possible implications of research uncertainty, and support initial quick-scan 'narratives' on research priorities (for example, possible implications of a climate sensitivity of $\left.5^{\circ} \mathrm{C}\right)$.

\section{Online content}

Any methods, additional references, Nature Research reporting summaries, source data, extended data, supplementary information, acknowledgements, peer review information; details of author contributions and competing interests; and statements of data and code availability are available at https://doi.org/10.1038/s41558-0200732-1.

Received: 28 February 2019; Accepted: 12 February 2020; Published online: 23 March 2020

\section{References}

1. IPCC. Climate Change 2014: Synthesis Report (eds Core Writing Team, Pachauri, R. K. \& Meyer, L. A.) (IPCC, 2014).

2. Clarke, L. et al. in Climate Change 2014: Mitigation of Climate Change (eds Edenhofer, O. et al.) 414-510 (Cambridge Univ. Press, 2014).

3. Edenhofer, O. et al. The economics of low stabilization: model comparison of mitigation strategies and costs. Energy J. 31, 11-48 (2010).

4. Riahi, K. et al. The Shared Socioeconomic Pathways and their energy, land use, and greenhouse gas emissions implications: an overview. Global Environ. Chang. 42, 153-168 (2017).

5. Rogelj, J. et al. Scenarios towards limiting global mean temperature increase below $1.5^{\circ} \mathrm{C}$. Nat. Clim. Change 8, 325-332 (2018).

6. Friedlingstein, P. et al. Climate-carbon cycle feedback analysis: Results from the C4MIP model intercomparison. J. Clim. 19, 3337-3353 (2006).

7. Collins, M. et al. in Climate Change 2013: The Physical Science Basis (eds Stocker, T. F. et al.) 1029-1136 (Cambridge Univ. Press, 2013).

8. Millar, R. J. et al. Emission budgets and pathways consistent with limiting warming to $1.5^{\circ} \mathrm{C}$. Nat. Geosci. 10, 741-747 (2017).

9. Schweizer, V. A few scenarios still do not fit all. Nat. Clim. Change 8 , 361-362 (2018).

10. Rozenberg, J., Guivarch, C., Lempert, R. \& Hallegatte, S. Building SSPs for climate policy analysis: a scenario elicitation methodology to map the space of possible future challenges to mitigation and adaptation. Clim. Change 122, 509-522 (2014)

11. Guivarch, C., Lempert, R. \& Trutnevyte, E. Scenario techniques for energy and environmental research: an overview of recent developments to broaden the capacity to deal with complexity and uncertainty. Environ. Modell. Softw. 97, 201-210 (2017).

12. Van Vuuren, D. P. et al. Stabilizing greenhouse gas concentrations at low levels: an assessment of reduction strategies and costs. Clim. Change 81, 119-159 (2007)

13. Rogelj, J., McCollum, D. L., Reisinger, A., Meinshausen, M. \& Riahi, K. Probabilistic cost estimates for climate change mitigation. Nature 493, 79-83 (2013).

14. Meinshausen, M. et al. Greenhouse-gas emission targets for limiting global warming to $2^{\circ} \mathrm{C}$. Nature 458, 1158-1162 (2009).

15. Gillett, N. P., Arora, V. K., Matthews, D. \& Allen, M. R. Constraining the ratio of global warming to cumulative $\mathrm{CO}_{2}$ emissions using CMIP5 simulations. J. Clim. 26, 6844-6858 (2013).

16. Booth, B. B. B. et al. High sensitivity of future global warming to land carbon cycle processes. Environ. Res. Lett. 7, 024002 (2012).

17. Visser, H., Dangendorf, S., Van Vuuren, D. P., Bregman, B. \& Petersen, A. C. Signal detection in global mean temperatures after 'Paris': an uncertainty and sensitivity analysis. Clim. Past 14, 139-155 (2018).

18. IPCC. Special Report on Global Warming of $1.5^{\circ} \mathrm{C}$ (eds Masson-Delmotte, V. et al.) (WMO, 2018).

19. van Vuuren, D. P. et al. Comparison of top-down and bottom-up estimates of sectoral and regional greenhouse gas emission reduction potentials. Energy Policy 37, 5125-5139 (2009).

20. Pollitt, H. \& Mercure, J. F. The role of money and the financial sector in energy-economy models used for assessing climate and energy policy. Clim. Policy 18, 184-197 (2017).

21. Kriegler, E. et al. The role of technology for achieving climate policy objectives: overview of the EMF 27 study on global technology and climate policy strategies. Clim. Change 123, 353-367 (2014).

22. Luderer, G. et al. Residual fossil $\mathrm{CO}_{2}$ emissions in $1.5-2{ }^{\circ} \mathrm{C}$ pathways. Nat. Clim. Change 8, 626-633 (2018).

23. Millar, R. J. \& Friedlingstein, P. The utility of the historical record for assessing the transient climate response to cumulative emissions. Phil. Trans. R. Soc. A 376, 20160449 (2018).

24. Butler, M. P., Reed, P. M., Fisher-Vanden, K., Keller, K. \& Wagener, T. Identifying parametric controls and dependencies in integrated assessment models using global sensitivity analysis. Environ. Modell. Softw. 59, 10-29 (2014).

25. Lemoine, D. \& McJeon, H. C. Trapped between two tails: trading off scientific uncertainties via climate targets. Environ. Res. Lett. 8, 034019 (2013).

26. Lamontagne, J. R., Reed, P. M., Marangoni, G., Keller, K. \& Garner, G. G. Robust abatement pathways to tolerable climate futures require immediate global action. Nat. Clim. Change 9, 290-294 (2019). 
27. Gillingham, K. et al. Modeling uncertainty in integrated assessment of climate change: a multimodel comparison. J. Assoc. Environ. Resour. Econ. 5, 791-826 (2018).

28. Sutton, R. T. ESD ideas: a simple proposal to improve the contribution of IPCC WGI to the assessment and communication of climate change risks. Earth Syst. Dynam. 9, 1155-1158 (2018).
29. IPCC. Climate Change 2013: The Physical Science Basis (eds Stocker, T. F. et al.) (Cambridge Univ. Press, 2013).

Publisher's note Springer Nature remains neutral with regard to jurisdictional claims in published maps and institutional affiliations.

(c) The Author(s), under exclusive licence to Springer Nature Limited 2020 


\section{Methods}

Analytical model. The metamodel used in this paper describes the mitigation costs for reaching global mean temperature targets as a function of uncertainty in the climate system, the uncertainty in the estimate for 2010 temperature, the non- $\mathrm{CO}_{2}$ forcing and the costs of reducing $\mathrm{CO}_{2}$ emissions.

The geophysical component of the metamodel used throughout this paper assumes a linear relation between cumulative $\mathrm{CO}_{2}$ emissions and the transient temperature change ${ }^{30}$. The temperature sensitivity is quantified through the TCRE. This parameter includes the mean effect of both $\mathrm{CO}_{2}$ and non- $\mathrm{CO}_{2}$ emissions to the total forcing. However, the TCRE distribution as estimated in the IPCC AR5 report does not cover the uncertainty in non- $\mathrm{CO}_{2}$ emissions. Accounting for this can be done using an extra term in the relationship between temperature and emissions (equation (2)), although alternative formulations are also possible (see below and SI). It is important to note that the forcing of non- $\mathrm{CO}_{2}$ emissions strongly correlates with cumulative $\mathrm{CO}_{2}$ emissions, while the range in forcing outcomes is constant for a wide range of cumulative $\mathrm{CO}_{2}$ emissions (Fig. 1b). This allows us to include a single constant factor $\sigma_{\text {non- }-\mathrm{CO}_{2}}$, with zero mean and a standard deviation equal to the uncertainty in Fig. 1b. The geophysical component of the model therefore becomes:

$$
T=T_{2010}+\operatorname{TCRE} \times \mathrm{CumCO}_{2}+\sigma_{\text {non- }-\mathrm{CO}_{2},}
$$

where $\mathrm{CumCO}_{2}$ is the cumulative emission $\int_{2010}^{2100} \mathrm{emis}(t) \mathrm{d} t$ and $T_{2010}$ the temperature in 2010.

The mitigation costs component of the metamodel is parametrized by an exponential relationship with the cumulative emissions, with the uncertainty captured in a single parameter $p$ :

$$
\text { costs }=\operatorname{costs}\left(\int_{2010}^{2100} \mathrm{emis}(t) \mathrm{d} t ; p\right) .
$$

By solving for the cumulative emissions, we obtain the full metamodel:

$$
\operatorname{costs}(T)=\operatorname{costs}\left(\frac{T-T_{2010}-\sigma_{\text {non- }-\mathrm{CO}_{2}}}{\mathrm{TCRE}} ; p\right) .
$$

Alternative model formulations. Equation (2) is based on the observed relationship between temperature increase and cumulative $\mathrm{CO}_{2}$ emissions for the RCP scenarios (TCRE), superimposing the contribution of non- $\mathrm{CO}_{2}$ gases as an uncertainty term that represents the wider non- $\mathrm{CO}_{2}$ forcing range in the full literature compared with the default relationship found for the RCPs $\left(\sigma_{\text {non-CO2 } 2}\right)$. This simple model (used in this paper) thus assumes a linear relationship between $\mathrm{CO}_{2}$ and non- $\mathrm{CO}_{2}$ forcing, with a constant uncertainty range around the default value (as indicated in Fig. 1b). An alternative, equivalent model would use a (lower) TCRE value for $\mathrm{CO}_{2}$-only scenarios and add a positive non- $\mathrm{CO}_{2}$ forcing term. The advantage of the latter model is that it can easily be adapted to different representations for the non- $\mathrm{CO}_{2}$ forcing as a function of cumulative $\mathrm{CO}_{2}$ (for example, introducing a minimum non- $\mathrm{CO}_{2}$ forcing term as a result of hardto-abate emission sources). Different models are tested in the Supplementary Information (the SI provides equations and results; see first discussion). As the results were found to be similar (Supplementary Table 1), we used the simplest model, that is, as represented by equation (2).

Model parameters. The distribution of the four parameters used in the metamodel (TCRE, $T_{2010}, \sigma_{\text {non- }-\mathrm{CO}_{2}}$ and $p$ ) are fully derived from currently available literature as used in key assessments focusing on multi-model outcomes. This means that the metamodel is able to go beyond the simple parametric uncertainty ranges of single models and also captures more structural differences across models. In the calculations, we base the range of each parameter on the literature range.

TCRE. The TCRE values are obtained directly from the uncertainty ranges indicated in the IPCC AR5 report ${ }^{1}$, starting from the pre-industrial values. In the IPCC calculations in AR5 and, more recently, in the special report on the $1.5^{\circ} \mathrm{C}$ target, it is assumed that the TCRE distribution is symmetric and normal. Interestingly, the underlying data (with a limited number of data points) suggests that the uncertainty range could be asymmetric. In combination with the fact that the TCRE is generally assumed to be strictly positive, it is possible to represent this using a $\beta$-PERT distribution ${ }^{31}$. Here, we tested both models and found that the model using an asymmetric TCRE distribution increases the carbon budget by $3 \%$ and decreases the mitigation costs by around 3\%. Because the exact form of the probability distribution of TCRE is unknown, here we decided to stick to the IPCC assumption for consistency and used a symmetric distribution.

By choosing the distribution parameters such that the TCRE best represents the AR5 pink plume, we obtain the following normal distribution:

$$
\mathrm{TCRE} \sim \mathcal{N}(0.62,0.12),
$$

where TCRE is expressed in ${ }^{\circ} \mathrm{C}$ per $\mathrm{TtCO}_{2}$. These numbers are similar to those reported in the literature, that is, $\mathrm{TCRE}$ of $0.2-0.7^{\circ} \mathrm{C}$ per $\mathrm{TtCO}_{2}$ (ref. ${ }^{7}$ ) and $0.23-0.66^{\circ} \mathrm{C}$ per $\mathrm{TtCO}_{2}$ (ref. ${ }^{15}$ ).
The information presented by the IPCC on the relationship between cumulative $\mathrm{CO}_{2}$ emissions and temperature increase (represented in Fig. 1a) allows the 5-95th percentile range for TCRE to be estimated. In AR5, the formal 5-95th percentile range of model results is assumed to represent the 16-84th interval (one s.d.) in order to represent the possibility that the models do not cover the full uncertainty range. In the SI, we tested both interpretations of the range in model outcomes (see the section on comparison of model outcomes with IPCC SR1.5 results). In the main article we aim to compare the impact of different uncertainty factors with the overall outcomes. As for each factor (mitigation costs, TCRE, non- $\mathrm{CO}_{2}$ forcing and 2010 temperature) one could argue that current models do not adequately represent the true uncertainty, we aim to use a similar method for all factors. Therefore, for all factors we used only the observed range in model outcomes (without ex-post adjustments).

Reference temperature. Second, the reference temperature $T_{2010}$ is also obtained from current literature using a set of independent datasets for the increase in global mean temperature ${ }^{17}$, giving the normal distributed parameter:

$$
T_{2010} \sim \mathcal{N}(0.909,0.075)
$$

where $T_{2010}$ is expressed in ${ }^{\circ} \mathrm{C}$.

The impact of non- $\mathrm{CO}_{2}$ gases on temperature. Third, the distribution of $\sigma_{\text {non- }-\mathrm{CO}_{2}}$ is obtained from the SSP database ${ }^{4,5}$. While this database only reports the forcing for $\mathrm{CO}_{2}$ and non- $\mathrm{CO}_{2}$ separately, the forcing can be converted to long-term temperature increases using the relation as used in the MAGICC climate model ${ }^{32}$. As shown in Fig. 1b, the non- $\mathrm{CO}_{2}$ temperature change contribution exhibits an almost linear behaviour as function of the cumulative $\mathrm{CO}_{2}$ emissions for each scenario in the SSP database. Moreover, as mentioned before, the variability is almost constant as a function of cumulative $\mathrm{CO}_{2}$ emissions. Using an ordinary least squares regression, we calculate this variability as the mean squared error of the residuals. This yields a normal distribution:

$$
\sigma_{\text {non }-\mathrm{CO}_{2}} \sim \mathcal{N}(0,0.121) .
$$

Again, the results are expressed in ${ }^{\circ} \mathrm{C}$.

Mitigation costs. Finally, the mitigation costs are also obtained from the SSP database. In the literature, various costs metrics are used, partly because the models can only express certain cost types. Three costs measures often used (and included in the SSP database) are: (1) consumption losses, (2) abatement costs-often measured as the area under an abatement curve-and (3) carbon prices. From the carbon price, abatement costs can be estimated by multiplying the carbon price and avoided $\mathrm{CO}_{2}$ emissions and a constant, and sum these over the 2010-2100 period (basically making an ex-post estimate of the area under abatement curve) (we used a value for the constant of 0.4, but its exact value is irrelevant as we only looked at relative costs). We have used these different metrics to include most data points as well as a wide range of possible models in the SSP database. However, the results were used following a preference order: if available for a scenario, consumption losses were used; if not, preferably reported abatement costs were used if available; only if both were not available, the metric based on carbon price and reduction volume was used. In each case, we calculated the net present value of the cumulative costs across the century using a 5\% discount rate. The latter is based on the notion that nearly all integrated assessment models used for the SSP database use a discount rate of $5 \%$ per year ${ }^{33}$. The results for the different models are shown in Supplementary Fig. 12. Subsequently, they have been transformed to a cost index: for each metric, the cost values are divided by their average cost of achieving a $1.6 \mathrm{TtCO}_{2}$ carbon budget. The resulting values are shown as dots in Fig. 1c. The figure shows that the relative increase in costs for the different costs metrics across the climate targets is very similar. Therefore, a single exponential curve has been fitted to these points using the data of all metrics. We assume that any carbon budget above $5.5 \mathrm{TtCO}_{2}$ can be reached without making any mitigation costs. The parametrized form of the costs is chosen to be:

$$
\operatorname{costs}\left(\mathrm{CumCO}_{2} ; a, b\right)=\left\{\begin{array}{ll}
a e^{-b \mathrm{CO}_{2}}-a e^{-5.5 b} & \mathrm{CumCO}_{2}<5.5 \\
0 & \mathrm{CumCO}_{2} \geq 5.5
\end{array},\right.
$$

where the parameters $a$ and $b$ are determined using the SSP data. Specifically, the above function is fitted through the minimum of the indexed abatement costs points, giving $a_{\min }=0.89$ and $b_{\min }=3.48$. Similarly, it is fitted through the maximum of the indexed points, giving $a_{\max }=15.01$ and $b_{\max }=0.55$. To capture the full uncertainty of the mitigation costs in a single parameter, and not in both $a$ and $b$, we define the parameter $p$, with values between 0 and 1 , used to calculate the linear combination of costs $\left(\mathrm{CumCO}_{2} ; a_{\min }, b_{\min }\right)$ and costs $\left(\mathrm{CumCO}_{2} ; a_{\max }, b_{\max }\right)$ :

$\operatorname{costs}\left(\mathrm{CumCO}_{2} ; p\right)=\operatorname{costs}\left(a_{\min }, b_{\min }\right)+p\left[\operatorname{costs}\left(a_{\max }, b_{\max }\right)-\operatorname{costs}\left(a_{\min }, b_{\min }\right)\right]$,

where we used $\operatorname{costs}(a, b)$ instead of $\operatorname{costs}\left(\mathrm{CumCO}_{2} ; a, b\right)$ for notational purposes.

Due to the asymmetry of the cost uncertainty, and the restriction that $p$ should take strictly positive values, the log-normal distribution is used. Since this 
distribution has a tail with too much weight than present in the SSP cost data, we truncate the distribution at $p=1.5$ :

$$
p \sim \log \operatorname{normal}(\mu=\ln [0.20615], \sigma=0.83555, \text { truncated at } 1.5),
$$

with $\mu$ and $\sigma$ being the mean and variance of the underlying distribution. More details and information about the exact fitting methods for each parameter can be found in ref. ${ }^{34}$.

To interpret the magnitude of the abatement costs, the cost index is expressed in US $\$$ by multiplying the cost index by the median net present value for consumption losses of the SSP scenarios with a carbon budget between 0.95 and $1.55 \mathrm{TtCO}_{2}$.

The distributions are therefore all assumed to be normal or log-normal. The reason is that each of the factors used in the metamodel is influenced by a range of underlying parameters that are mostly independent. For instance, the TCRE range is a function of the $\mathrm{CO}_{2}$ forcing, ocean heat uptake, carbon cycle feedbacks and several other factors. Relative abatement costs are a function of the costs uncertainty of individual technologies, the degree of participation of sectors and countries in climate policy, learning rates, preferences, and so on. While each unique combination of values for the underlying parameters could be equally likely, the full distribution of all combinations is likely to follow a representation close to a normal or log-normal distribution based on the central limit theorem. We have tested for the non- $\mathrm{CO}_{2}$ forcing and relative costs whether the distribution could be consistent with the assumed distribution using a Q-Q plot (see SI. D). It was found that the assumed distribution was indeed consistent with the data.

Monte Carlo sampling. We use a sampling method to obtain the model result since it is not possible to calculate the resulting distribution of the costs analytically, given the four input parameters and their distributions, Specifically, for a range of values of $\mathrm{CO}_{2}$, we sample a total of $N$ values from the distributions of TCRE, $T_{2010}, \sigma_{\text {non- }-\mathrm{CO}_{2}}$ and $p$, and separately calculate the resulting temperature using equation (2) and the abatement costs using equation (3). The resulting point has then as abscissa the temperature, and as ordinate the costs. This is shown in Fig. $2 \mathrm{a}, \mathrm{c}, \mathrm{d}$ for 6,500 values of $\mathrm{CO}_{2}$ between 0.5 and $6 \mathrm{TtCO}_{2}$. It should be noted that we could also have sampled without the separation step using the $\mathrm{CO}_{2}$ with equation (4). In fact, by choosing $N$ values of the temperature $T$, and sampling the parameter distributions for every point, we directly obtain the empirical distribution of the costs as a function of temperature. We do not use the outcomes of the model for values lower than $0.5 \mathrm{TtCO}_{2}$. The reason is that most of the empirical data, especially on costs, are available only for cumulative emissions above this level. Using the model for lower values would therefore lead us outside the realm of expert model runs. Therefore, in Fig. 2d, that there is a smaller point density towards lower temperature goals is not just because of the higher variance in costs, but also since we exclude samples with $\mathrm{CO}_{2}$ values lower than $0.5 \mathrm{TtCO}_{2}$.

Carbon budgets. The carbon budgets in Fig. $2 \mathrm{~b}$ are calculated as the cumulative emissions from 2010 to 2100 such that $66 \%$ of the sampled values of the temperature of equation (2) are below or equal to a chosen target temperature.

Partial uncertainty estimation. The partial uncertainties are estimated using variance-based sensitivity analysis ${ }^{35}$. Let $Y$ be a random variable with input variables $X_{i}, \ldots, X_{n}$, in our case, TCRE, $T_{2010}, \sigma_{\text {non- }-\mathrm{CO}_{2}}$ and $p$. The total variance $\operatorname{Var}(Y)$ is decomposed into a sum of partial variances (variances of the expectation value of $Y$ conditional on the set of variables $X$ ):

$$
\operatorname{Var}(Y)=\sum_{i=1}^{n} V_{i}+\sum_{i<j} V_{i j}+\cdots+V_{12 \ldots n},
$$

where

$$
\begin{gathered}
V_{i}:=\operatorname{Var}_{X_{i}}\left(\mathbb{E}\left[Y \mid X_{i}\right]\right), \\
V_{i j}:=\operatorname{Var}_{X_{i j}}\left(\mathbb{E}\left[Y \mid X_{i}, X_{j}\right]\right)-V_{i}-V_{j} .
\end{gathered}
$$

These conditional variances are then converted to relative partial variance by dividing them by the total variance $\operatorname{Var}(Y)$. In our analysis, we only consider the first order terms $V_{i}$ and ignore the higher order interaction terms. The second and third order terms $V_{i j}$ and $V_{i j k}$ are shown in the SI. The variance terms are estimated using a Monte Carlo method ${ }^{36}$. For each temperature value, we sample one million values from each distribution, which are then used as input for the Monte Carlo method. The calculations are then repeated 50 times and averaged for increased accuracy.

\section{Data availability}

The data used to assess the impact of non- $\mathrm{CO}_{2}$ emissions and the mitigation costs that support the findings of this study are publicly available online at the scenario databases hosted by IIASA: https://tntcat.iiasa.ac.at/SspDb/dsd (ref. ${ }^{4}$ ), https:// tntcat.iiasa.ac.at/AR5DB/dsd (ref. ${ }^{2}$ ) and https://data.ene.iiasa.ac.at/iamc-1.5cexplorer/\#/workspaces $\left(\right.$ ref. ${ }^{18}$ ). The data for each figure are available at the PBL/IMAGE website.

\section{Code availability}

Model codes are available at https://github.com/kvanderwijst/ variancedecomposition-IAM (https://doi.org/10.5281/zenodo.3633944).

\section{References}

30. Rogelj, J. et al. Differences between carbon budget estimates unravelled. Nat. Clim. Change 6, 245-252 (2016).

31. Vose, D. Risk Analysis: A Quantitative Guide (John Wiley \& Sons, 2008).

32. Meinshausen, M., Raper, S. C. B. \& Wigley, T. M. L. Emulating coupled atmosphere-ocean and carbon cycle models with a simpler model, MAGICC6 - Part 1: model description and calibration. Atmos. Chem. Phys 11, 1417-1456 (2011)

33. The common Integrated Assessment Model (IAM) documentation. IAMC https://www.iamcdocumentation.eu/index.php/IAMC wiki (2018).

34. Wijst, K.-I. v. d. Optimal Policy for Carbon Pricing: Challenging the Hotelling Rule and Dissecting Mitigation Cost Uncertainties. Master of Mathematical Sciences thesis, Utrecht Univ. (2018).

35. Sobol, I. M. On sensitivity estimation for nonlinear mathematical models. Matem. Mod. 2, 112-118 (1990).

36. Saltelli, A. Making best use of model evaluations to compute sensitivity indices. Comput. Phys. Commun. 145, 280-297 (2002).

\section{Acknowledgements}

The research presented in this paper benefitted from funding under the European Union's Horizon 2020 Framework Programme for Research and Innovation under grant agreement nos. 82112 (NAVIGATE) and 641816 (CRESCENDO). In addition, C.J. was supported by the Joint UK BEIS/Defra Met Office Hadley Centre Climate Programme (grant no. GA01101).

\section{Author contributions}

D.P.v.V., K.-I.v.d.W. and S.M. designed the metamodel and the experiments. All authors contributed to the elaboration of the model and the writing of the article.

\section{Competing interests}

The authors declare no competing interests.

\section{Additional information}

Supplementary information is available for this paper at https://doi.org/10.1038/ s41558-020-0732-1.

Correspondence and requests for materials should be addressed to D.P.v.V.

Peer review information Nature Climate Change thanks James Glynn and the other, anonymous, reviewer(s) for their contribution to the peer review of this work.

Reprints and permissions information is available at www.nature.com/reprints. 